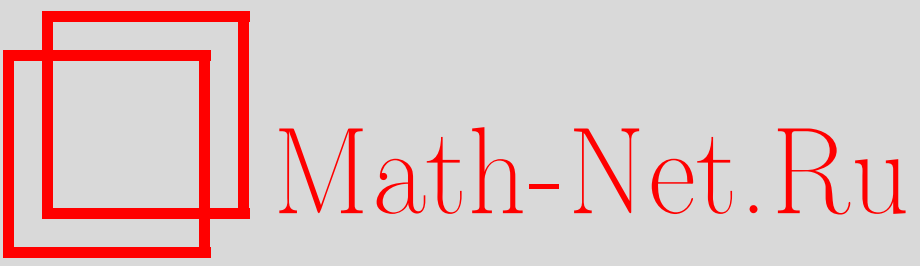

А. А. Годин, Структура идеалов полиномиальных почтиколец и их факторколец, УМH, 2001, том 56, выпуск 6, 143-144

DOI: https://doi.org/10.4213/rm458

Использование Общероссийского математического портала Math-Net.Ru подразумевает, что вы прочитали и согласны с пользовательским соглашением

http://www.mathnet.ru/rus/agreement

Параметры загрузки:

IP : 54.174 .149 .18

26 апреля 2023 г., 15:41:28 


\title{
СТРУКТУРА ИДЕАЛОВ ПОЛИНОМИАЛЬНЫХ ПОЧТИКОЛЕЦ И ИХ ФАКТОРКОЛЕЦ
}

\author{
А. А. Годин
}

Пусть $\mathrm{R}$ - кольцо. Пока не сказано обратное, $\mathrm{R}$ предполагается коммутативным. Пусть $\mathrm{R}[x]$ - кольцо многочленов от переменной $x$, a $\mathrm{NR}[x]$ - почтиколццо многочленов от переменной $x$. Напомним, что умножение в $\mathrm{NR}[x]$ задается композицией многочленов $[1]$.

Положим $\mathrm{R}_{0}[x]:=\{p \in \mathrm{R}[x] \mid p(0)=0\}, \mathrm{NR}_{0}[x]:=(\mathrm{NR}[x])_{0}, \mathrm{NR}_{0, n}[x]:=\mathrm{NR}_{0}[x] /\left\langle x^{n}\right\rangle$, $\mathrm{R}_{0, n}[x]:=\mathrm{R}_{0}[x] /\left\langle x^{n}\right\rangle$. Рассмотрим связь между идеалами кольца многочленов и левыми идеалами соответствующего почтикольца.

ЛЕмма 1. Все идеалы кольца многочленов $\mathrm{R}_{0}[x]$ являются левыми идеалами соответствующего почтикольца $\mathrm{NR}_{0}[x]$.

Введем следующие обозначения:

$I \triangleleft_{l} R: I$ - левый идеал (почти-)кольца $\mathrm{R}$;

$I \triangleleft_{r} R: I$ - правый идеал (почти-)кольца $\mathrm{R}$;

$I \triangleleft R: I$ - двусторонний идеал (почти-)кольц $\mathrm{R}$.

Теорема 1. Пусть $\mathrm{R}-2$-делимое кольцо. Тогда:

1) $I \triangleleft_{l} \mathrm{NR}_{0}[x] \Longleftrightarrow I \triangleleft \mathrm{R}_{0}[x]$;

2) двухсторонние идеаль почтикольца $\mathrm{NR}_{0}[x]$ - это идеаль кольца $\mathrm{R}_{0}[x]$, выдерживающие подстановку.

ЗАмечание 1. Если $\mathrm{R}$ - не 2-делимое кольцо, то существует идеал в $\mathrm{NR}_{0}[x]$, не являющийся идеалом в $\mathrm{R}_{0}[x]$.

Пусть $\mathrm{R}$ - поле, $\operatorname{char} \mathrm{R} \neq 2$.

ТЕОрема 2. Все идеаль почтикольца $\mathrm{NR}_{0, n}$ исчерпьваются идеалами кольца $\mathrm{R}_{0, n}$, порожденными $x^{k}$ для подходящего $k$.

ЗАмЕЧАНИЕ 2. В случае произвольного конечного поля $\mathrm{R}$ утверждение предыдущей теоремы неверно.

Пусть $\mathrm{R}$ - коммутативное кольцо (не обязательно 2-делимое). Определим структуру почтикольца многочленов от нескольких переменных следующим образом.

Пусть $\mathfrak{R}_{n}^{\prime}=\mathrm{R}\left[x_{1}, \ldots, x_{n}\right]$ - кольцо многочленов от $n$ переменных. Рассмотрим $\mathfrak{R}_{n}^{\prime n}$ и $\mathrm{N} \mathfrak{R}_{n}^{\prime}=$ $\mathrm{NR}\left[x_{1}, \ldots, x_{n}\right]=\left\{\mathfrak{R}_{n}^{\prime n},+, \circ\right\}$, где

$$
\left(\begin{array}{c}
p_{1} \\
\vdots \\
p_{n}
\end{array}\right) \circ\left(\begin{array}{c}
q_{1} \\
\vdots \\
q_{n}
\end{array}\right)=\left(\begin{array}{c}
p_{1}\left(q_{1}, \ldots, q_{n}\right) \\
\vdots \\
p_{n}\left(q_{1}, \ldots, q_{n}\right)
\end{array}\right) .
$$

Определим $\mathfrak{R}_{n}:=\left\{P \in \mathfrak{R}_{n}^{\prime n} \mid P(0)=0\right\}$ и $\mathrm{N} \mathfrak{R}_{n}:=\left(\mathrm{N} \mathfrak{R}_{n}^{\prime}\right)_{0}$ Легко видеть, что $\mathrm{N} \mathfrak{R}_{n}$ и $\mathrm{N} \mathfrak{R}_{n}^{\prime}-$ почтиколшца, а $\left\{\mathrm{N} \mathfrak{R}_{n},+, \circ, \cdot\right\}$ и $\left\{\mathrm{N} \mathfrak{R}_{n}^{\prime},+, \circ, \cdot\right\}$ - композиционные колшца.

ТЕОРема 3. Пусть $\mathrm{R}-$ произвольное кольцо, $n \geqslant 2$. Тогда:

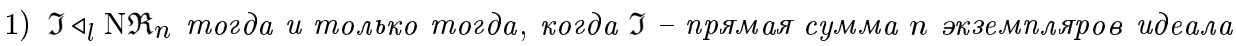
$I \triangleleft \Re_{n} ;$

2) $\mathfrak{I} \triangleleft \mathrm{N} \mathfrak{R}_{n}$ тогда и только тогда, когда $\mathfrak{I}$ - прямая сумма $п$ әкземпляров идеала $I \triangleleft \Re_{n}$, выдерживающего подстановки.

Пусть R - поле.

Теорема 4. Если поле $\mathrm{R}-$ - бесконечно, $n \geqslant 2$ или char $\mathrm{R} \neq 2$, то идеальи почтикольца $\mathrm{N} \Re_{n}-$ мономиальны. 
СледСтвиЕ. Если $\mathrm{R}$ - бесконечно и char $\mathrm{R} \neq 2$, то все идеаль почтикольца $\mathrm{NR}_{0}[x]$ порождены $x^{k}$ для подходящего $k$.

Теорема 5. Если $\mathrm{R}$ - бесконечно и $n \geqslant 2$, то $\mathrm{N}_{n}^{\prime}[n]$ просто.

Tеорема 6 [2]. Если $\mathrm{R}$ - бесконечно, $n \geqslant 2$ u char $\mathrm{R} \neq 2$, mо $\mathrm{N} \mathfrak{R}_{n}^{\prime}[x]$ просто.

Пусть $\mathfrak{S}_{n}$ - идеал в $\mathfrak{R}_{n}$, порожденный многочленами полной степени $n$.

Теорема 7. Если R - поле характеристики 0, то все идеаль $\mathrm{N} \Re_{n}-$ в точности прямые суммы $n$ әкземпляров идеалов $\mathfrak{S}_{k}$ для подходящего $k$.

ЗАмЕчАнИЕ 3 . В случае характеристики $p$ этот резултат неверен.

ЛЕмма 2. Пусть I - идеал, выдерживающий подстановки. Если D-степень минимального монома из $I$, mo $_{n} \mathfrak{S}_{n} \subset I$.

Заметим, что $\mathrm{N} \Re_{n} / \mathfrak{S}_{2}-$ кольцо, изоморфное $\mathrm{M}_{n}[\mathrm{R}]$.

Рассмотрим теперь некоммутативный случай и $n \geqslant 2$. Далее $\mathrm{R}-$ произвольное (не обязательно коммутативное) кольцо.

ОПРЕДЕЛЕНИЕ. Пусть $\mathbf{V}$ - некое многообразие колец и $\mathbf{R} \in \mathbf{V}$. Рассмотрим относительно свободное произведение $\mathfrak{R}_{n}=\mathrm{R} * \mathbf{V} F_{\mathbf{V}}\left(x_{1}, \ldots, x_{n}\right)$, где $F_{\mathbf{V}}\left(x_{1}, \ldots, x_{n}\right)$ - относительно свободная алгебра от образующих $x_{1}, \ldots, x_{n}$.

ЗАмечАниЕ 4 . Заметим, что если $\mathbf{V}=A b$ - многообразие коммутативных колец, то наше определение дает "обычное" кольцо многочленов от переменных $x_{1}, \ldots, x_{n}$.

Определение почтикольца $\mathrm{N} \Re_{n}$ остается прежним, т.е. почтикольцом вектор-многочленов с покомпонентным сложением и композицией, заданной формулой (1). Аналогично определим кольцо $\Re_{n}^{n}$.

Теорема 8. Пусть $\mathrm{R}$ - произвольное кольцо. Тогда:

1) $\mathfrak{I} \triangleleft_{l} \mathrm{~N} \Re_{n}$ тогда и только тогда, когда $\mathfrak{I}$ - прямая сумма п әкземпляров идеала $I \triangleleft \Re_{n}$

2) двусторонние идеалы почтикольиа $\mathrm{N} \mathfrak{R}_{n}$ - в точности прямые произведения $n$ экземпляров идеала $I \triangleleft \Re_{n}$, выдерживающего подстановку (T-идеала).

Перейдем от конечной прямой суммы к бесконечной. Пусть $\mathfrak{R} \infty-$ кольцо многочленов от бесконечного числа переменных (прямой предел колец $\mathfrak{R}_{n}, n \rightarrow \infty$ ). Тогда определим почтикольцо $\mathrm{N} \Re_{\infty}$ как прямую сумму счетного числа $\mathfrak{R}_{\infty}$ с покомпонентным сложением и композицией о, определяемой так же, как и в $\mathrm{N} \Re_{n}$. Корректность следует из определений прямой суммы

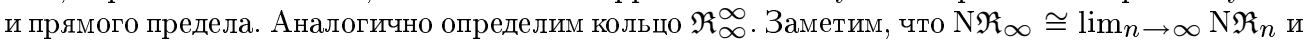
$\mathfrak{R}_{\infty}^{\infty} \cong \lim _{n \rightarrow \infty} \mathfrak{R}_{n}^{n}$.

Теорема 9. Пусть $\mathrm{R}-$ произвольное кольцо. Тогда:

1) $\mathfrak{I}_{\triangleleft_{l}} \mathrm{~N} \Re_{\infty}$ тогда и только тогда, когда $\mathfrak{I}$ - прямая сумма счетного числа әкземпляров идеала $I \triangleleft \Re_{\infty}$;

2) двусторонние идеаль $\mathfrak{I} \triangleleft \mathrm{N} \Re_{\infty}-$ в точности прямые произведения счетного числа экземпляров $T$-идеала $I \triangleleft \Re \infty$.

\section{СПИСОК ЛИТЕРАТУРЫ}

[1] J. D. P. Meldrum. Near-Rings and Their Links with Groups. Marshfield, MA: Pitman, 1985. [2] G.Pilz. Near-Rings. Amsterdam: North-Holland, 1983. 